\title{
Perspectives of Radio over Fiber Technologies
}

\author{
A.M.J. Koonen, M. García Larrodé, A. Ng'oma*, K. Wang, H. Yang, Y. Zheng, E. Tangdiongga \\ COBRA Institute, Dept. of Electrical Engineering, Eindhoven University of Technology, \\ Den Dolech 2, P. O. Box 513, NL 5600 MB Eindhoven, The Netherlands, e-mail: a.m.j.koonen@tue.nl \\ (* A.Ng'oma is now with Corning Inc., NY, USA)
}

\begin{abstract}
Radio-over-Fiber technologies enable efficient provisioning of broadband wireless services both in access and in in-building networks, in particular when combined with flexible optical routing and dispersion-robust RoF transport techniques, such as optical frequency multiplying.

(C)2008 Optical Society of America

OCIS codes: (060.2330) Fiber optics communications; (060.5625) Radio frequency photonics
\end{abstract}

\section{Introduction}

Wireless services are taking a steadily increasing share of the telecommunications market. The end users not only benefit from its main virtue, mobility, but are also demanding ever larger bandwidth. Larger wireless capacity per user requires the reduction of the wireless cell size, i.e. establishing pico-cells, which is enabled by increasing the radio microwave frequency. The wireless LAN IEEE $802.11 \mathrm{~g}$ WiFi standard offers up to $54 \mathrm{Mbit} / \mathrm{s}$ in the $2.5 \mathrm{GHz}$ range; the IEEE $802.16 \mathrm{WiMAX}$ standard offers up to $100 \mathrm{Mbit} / \mathrm{s}$ in the range of 10 to $66 \mathrm{GHz}$; IEEE 802.15 .3 UWB operates at frequencies up to $60 \mathrm{GHz}$, offering short-range capacity up to $480 \mathrm{Mbit} / \mathrm{s}$. Smaller radio cells imply that ever more antennas are needed to cover a certain area. Such an area may encompass the rooms in a residential home, but may also be a hospital, an office building, an airport lounge, a conference site, etc. When needing so many antenna sites, it becomes economically attractive to locate the microwave signal generation and modulation not at every antenna, but centrally in a cabinet from where optical fibers with their inherent low losses and wide bandwidth can nicely bring the signal to the antennas. The antennas then only have to do the simple optical-to-electrical conversion, and to emit and receive the wireless signal. Centralizing the sophisticated signal handling eases maintenance and upgrading. The signal handling may include techniques for multiple-inputmultiple-output antenna schemes, smart beam forming antennas, mobility and connection handovers, feeding multiple radio standards to an antenna, reconfiguration of services to antennas, etc. Hence radio-over-fiber (RoF) technologies can bring many advantages in operating, maintaining and upgrading wireless networks.

This paper addresses two major application areas of RoF: outdoor fixed wireless access via standard singlemode fiber, where the last link to the end user's premises is established wirelessly, and indoor wireless access via preferably multimode fiber (already widely installed in buildings), where flexible local networks need to be realized.

\section{Radio over Fiber technologies}

A lot of techniques have been reported to transport radio signals over fiber to the antenna site.

Basically, one may directly modulate the light intensity of the optical source by the radio signal. At the antenna station, the received optical signal just needs a photodetector and bandpass amplifier, and the regained radio signal is then to be radiated by the antenna. However, the bandwidth and linearity requirements on the laser transmitter and the receiver are high. Also careful fiber dispersion compensation techniques may be needed at higher RF frequencies and longer fiber lengths. Thus the application of this intensity-modulation (IM) scheme is restricted to the low/medium RF range.

Alternatively, one may use various optical frequency conversion methods to generate the microwave signal at the antenna site. As a first method, two narrow-linewidth laser diodes may be deployed, of which one is intensitymodulated with the data signal. When the optical frequency spacing between these two lasers is carefully kept equal to the desired microwave frequency, after traveling through the fiber link, the heterodyning of the two optical signals in the photodiode generates the modulated microwave carrier. However, the linewidth of the microwave signal is equal to the sum of the linewidths of the two laser diodes, and may exceed the requirements for adequate detection of the usually multilevel signal modulation format (such as multi-level QAM). Hence the linewidth of the laser diodes needs to be very small, which is e.g. achievable by injection locking.

A second optical frequency conversion method uses just a single laser followed by a Mach Zehnder Interferometer (MZI) modulator which is biased at its inflexion point $\left(\mathrm{V}_{\pi}\right)$ and is driven by a sinusoidal signal at half the desired microwave frequency [1]. At the MZI's output, a two-tone optical signal emerges with a tone spacing equal to the microwave frequency, and with suppressed optical carrier. When modulating the laser with the 
data signal, heterodyning in the photodiode at the receiver generates the modulated microwave signal at $f_{R F}$. This method suppresses the phase noise of the laser diode, and hence creates a clean microwave.

A third method deploys the generation of many harmonics by the so-called Optical Frequency Multiplying (OFM) technique. Its principle is shown in Fig. 1. The optical frequency of a wavelength-tunable laser diode in the headend station is periodically swept over an optical frequency range $\beta \cdot \omega_{m}$ with a harmonic sweep signal having a frequency $\omega_{m}$. After passing the fixed MZI, by FM-to-IM conversion many harmonics of the sweep frequency are generated. The strengths of the harmonics can be set by adjusting the frequency modulation index $\beta$. At the antenna site, when neglecting the fiber's attenuation and dispersion, the output current signal $i_{\text {out }}(t)$ of the photodiode contains all these harmonics $n \cdot \omega_{m}$ with $n$-th order Bessel function weight $J_{n}\left(2 \beta \cdot \sin \left(\omega_{m} \tau / 2\right)\right)$, according to [2]

$$
i_{\text {out }}(t)=I_{0} \cdot\left\{1+\cos \left[2 \beta \cdot \sin \left(\frac{1}{2} \omega_{s w} \tau\right) \cdot \cos \left(\omega_{s w}\left(t-\frac{1}{2} \tau\right)\right)+\omega_{0} \tau+\dot{\varphi} \tau\right]\right\}
$$

in which $I_{0}$ is the DC current proportional to the received optical power, $\tau$ is the delay difference in the MZI arms, $\omega_{0}$ is the central frequency of the laser diode, and $\dot{\varphi}=d \varphi(t) / d t$ is its phase noise.

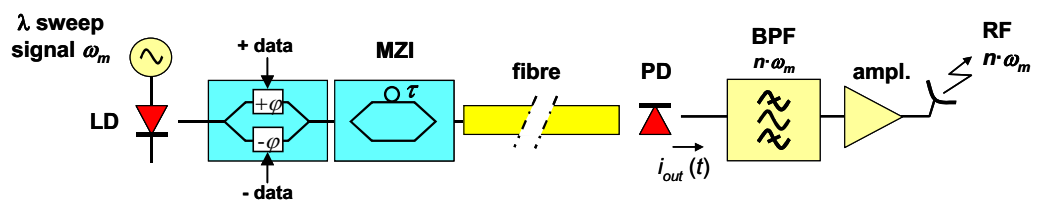

Fig. 1 Optical Frequency Multiplying

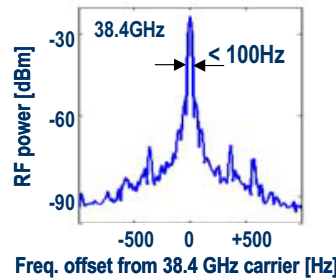

Fig. $238.4 \mathrm{GHz}$ microwave carrier generated by OFM

The data signal is present on all the harmonics. After photodetection, a bandpass filter extracts the desired harmonic (or several harmonics), which is amplified and emitted as a microwave carrier bearing the data signal. The laser phase noise has negligible impact if $\dot{\varphi} \tau<<\pi / 2$, i.e. when the laser linewidth is much smaller than a quarter of the Free Spectral Range $\Delta \omega_{F S R}=2 \pi / \tau$ of the MZI. This condition is easily met, as the MZI's FSR is typically around $10 \mathrm{GHz}$, and thus the OFM process effectively suppresses the laser phase noise. Hence very pure microwave signals can be generated. Linewidths below the measurement resolution $(<100 \mathrm{~Hz})$ have been obtained when generating a $38.4 \mathrm{GHz}$ carrier as the $6^{\text {th }}$ harmonic of a $6.4 \mathrm{GHz}$ sweep signal, whereas the linewidth of the laser diode was more than $1 \mathrm{MHz}$; see Fig. 2. Moreover, by using a high order harmonic, OFM permits to use only a relatively lowfrequency sweep generator in the headend, which can be of high quality while still at low cost.

\section{Radio over single-mode fiber in access networks}

Direct RF intensity modulation of a laser diode yields a double-sideband (IM-DSB) spectrum. Fiber dispersion causes phase shifts between the two sidebands, which leads to severe fading of the microwave at certain fiber lengths, as shown in Fig. 3 [3]. On the other hand, the OFM technique is very robust against chromatic dispersion in single-mode fiber, as shown also in Fig. 3 over $72 \mathrm{~km}$ of SMF. This dispersion-robustness is very useful when operating in link-switched networks, where the fiber connecting the headend to an antenna may vary in length.

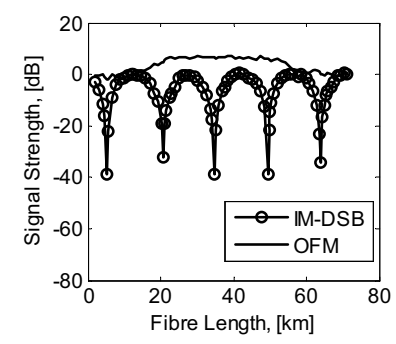

Fig. 3 Measured impact of SMF dispersion on relative strength of $30 \mathrm{GHz}$ carrier, using IM-DSB or OFM (excluding fiber losses)

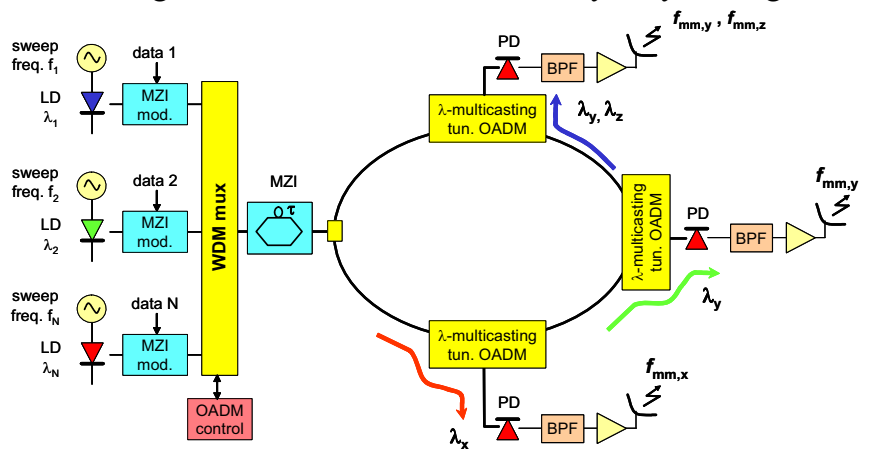

Fig. 4 Flexible routing of radio capacity in Fixed Wireless Access

Fig. 4 shows such a network where at the headend station different microwave signals are generated by OFM in different wavelength channels. Using routing by means of tunable wavelength add-drop modules, one or more of these wavelength channels can be dropped to an antenna site, and thus the radio capacity can be flexibly assigned among these antennas depending on the local traffic demands. Moreover, the frequency chirping of the source 


\section{OThP3.pdf}

which is inherent to the OFM principle yields an increase of the Stimulated Brillouin Scattering (SBS) threshold, and thus it enables to transport significantly higher optical power levels and generate stronger microwave signals from the antenna. Experimentally it was observed that the OFM system on a $25 \mathrm{~km}$ SMF link exhibited a much higher SBS threshold than the IM-DD system (i.c. $7 \mathrm{~dB}$ higher, from 10 to $50 \mathrm{~mW}$ ).

\section{Radio over multimode fiber in in-building networks}

Multimode (silica or plastic) optical fiber is widely used in in-building networks, due to its ease of installation. Its modal dispersion, however, is hampering radio signal transport. The OFM technique has shown to be robust against modal dispersion; it has been derived mathematically that its generated harmonics scale with the fiber's frequency characteristics. Thanks to the very pure microwave carrier generated with the OFM technique, transmission of 16-QAM and 64-QAM signals up to $120 \mathrm{Mbit} / \mathrm{s}$ in the $24-30 \mathrm{GHz}$ band has successfully been demonstrated over $4.4 \mathrm{~km}$ of silica $50 \mu \mathrm{m}$ core graded index fiber [4]. Using five subcarriers, over the same link simultaneous transmission of five 64-QAM signals with a total bitrate of $210 \mathrm{Mbit} / \mathrm{s}$ has been demonstrated [5]. Also a 100 $\mathrm{Mbit} / \mathrm{s}$ 16-QAM bidirectional system at $17.2 \mathrm{GHz}$ over $100 \mathrm{~m}$ of $50 \mu \mathrm{m}$ core graded-index POF has been shown, where for upstream transmission the microwave signal received at the antenna site is downconverted with the 17.2 GHz microwave carrier generated at the headend; see Fig. 5 [6]. When creating pico-cells for broadband wireless communication at high microwave frequencies, the required line-of-sight prevents communication between rooms. However, transparent transport of the microwave signals by means of radio-over-fiber techniques can establish communication between two or more rooms. Fig. 6 shows how with a transparent optical switch in the HCC (Home Communication Controller) rooms can flexibly be interconnected, and thus their pico-cells can be merged into a reconfigurable "wireless virtual private network", without interference with other wireless VPN-s. The remote antenna concept will add latency with respect to the conventional case where the wireless protocols are terminated in the base station at the antenna site. This will reduce the network's throughput. For centrally scheduled MAC protocols, such as WiMAX, it has been shown that this reduction will be only small $(<1 \%)$ when fiber link lengths are less than 500 metres.

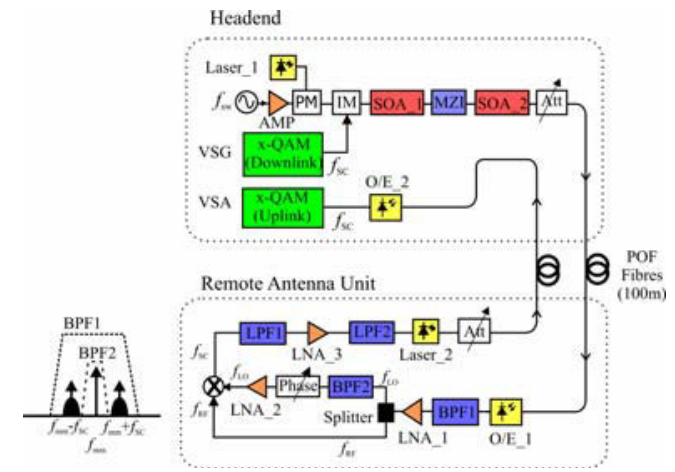

Fig. 5 16-QAM 100 Mbit/s $17.2 \mathrm{GHz}$ bi-directional radio-over-POF link

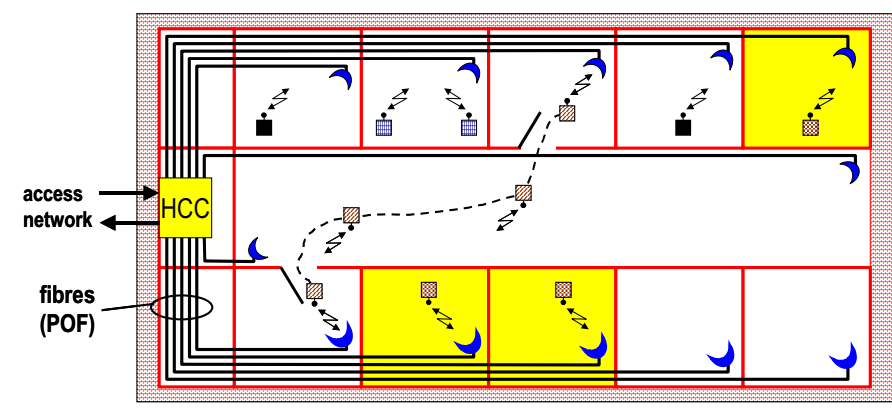

Fig. 6 Flexible inter-room wireless communication using switched RoF links

\section{Conclusions}

Radio-over-Fiber technologies can provide transport of broadband microwave signals for SMF and MMF based networks. Flexible optical routing and dispersion-robust transport techniques, such as Optical Frequency Multiplying, enable to improve the network's throughput and operational efficiency.

\section{References}

[1] R.A. Griffin, P.M. Lane, J.J. O’Reilly, “Radio-over-fibre distribution using an optical millimeter-wave/DWDM overlay”, Proc. OFC'99, San Diego, Feb. 22-25, 1999, paper WD6

[2] A.M.J. Koonen and A. Ngoma, "Integrated broadband optical fibre/ wireless LAN access networks," in Broadband Optical Access Networks and Fiber-to-the-Home: System Technologies and Development Strategies. New York: Wiley, 2006

[3] A. Ng'oma, G.-J. Rijckenberg, A.M.J. Koonen, "Building extended-recah radio-over-fiber links by exploiting Optical Frequency Multiplication's dispersion tolerance”, Proc. IEEE Int. Microwave Symp., Honolulu, June 3-8, 2007

[4] M. Garcia Larrode, A.M.J. Koonen, J.J. Vegas Olmos, "Transmission of microwave signals beyond the modal bandwidth of multimode fiber links", Proc. of Int. Top. Meeting on Microwave Phot., Grenoble, Oct. 3-6, 2006

[5] H. Yang, M. Garcia Larrode, G.-J. Rijckenberg, A. Ng'oma, E. Tangdiongga, A.M.J. Koonen, "Radio-over-fibre transmission of multi-carrier 64-QAM radio signal at $18 \mathrm{GHz}$, Proc. of BroadBand Europe, Antwerp, Dec. 3-6, 2007

[6] A. Ng'oma, J. Zeng, H.P.A. van den Boom, Y. Watanabe, A.M.J. Koonen, "Bi-Directional Polymer Optical Fiber-based System for Distributing 100 Mbps WiMAX Signals Exceeding 17 GHz", Proc. of AP-MWP2007, Jeju Island, South Korea, April 25-27, 2007 